\title{
SNS LONGITUDINAL SPACE CHARGE EFFECT
}

\section{BNL/SNS TECHNICAL NOTE}

NO. 043

S. Y. Zhang and W. T. Weng

April 6, 1998

ALTERNATING GRADIENT SYNCHROTRON DEPARTMENT

BROOKHAVEN NATIONAL LABORATORY

UPTON, NEW YORK 11973 


\section{Summary}

1. Using both analytical approach and simulation, it is found that for 2 MW SNS storage ring, the longitudinal space charge induced bunch leakage into the interbunch gap is not significant. Therefore, corrections to the longitudinal space charge impedance, such as the proposed ferrite insertion in the PSR ring, are probably not necessary.

2. Applying an RF voltage ramping from $20 \mathrm{KV}$ to $40 \mathrm{KV}$ during the multiturn injection can further cut the bunch leakage to negligible.

3. The same approaches applied to the PSR show that the longitudinal space charge effect does cause sizable bunch leakage at the intensity limit encountered there.

\section{Space Charge}

As usual, the longitudinal space charge impedance is defined as,

$$
Z_{S C}=-j \frac{Z_{0} g}{2 \beta \gamma^{2}}
$$

where $Z_{0}=377 \Omega$, and

$$
g=1+2 \log \left(\frac{b}{a}\right)
$$

with $a$ and $b$ being average radii of the beam and vacuum chamber, respectively. For SNS, the machine average vacuum chamber radius $b=0.1 \mathrm{~m}$ and the beam size $a=0.0236 \mathrm{~m}$ give rise to the longitudinal space charge impedance $Z_{S C}=-j 196 \Omega$.

For a beam current $I$, the space charge impedance induced voltage, a particle sees per turn, can be written as,

$$
V_{S C}=\frac{d I}{d \phi}\left|Z_{S C}\right|
$$

where $\phi$ is the beam phase deviation in radius. This voltage represents a longitudinal defocusing force at the SNS, reducing the effective RF voltage within the bunch duration. 
We first consider an analytical approach to the longitudinal space charge effect for the SNS. The space charge potential is defined using $V_{S C}$ as,

$$
U_{S C}\left(\phi_{\ell}\right)=\int_{0}^{\phi_{\ell}} V_{S C} d \phi=\int_{0}^{\phi_{\ell}} \frac{d I}{d \phi}\left|Z_{S C}\right| d \phi
$$

where $\phi_{\ell}$ is the half bunch length in radius. The RF voltage potential, with second $R F$, at the bunch length $\phi_{\ell}$ is,

$$
U_{R F}\left(\phi_{\ell}\right)=\int_{0}^{\phi_{\ell}} V(\sin \phi-0.5 \sin 2 \phi) d \phi
$$

Whether the bunch leaks into the interbunch gap or not is determined by two factors. The first factor is the particle maximum momentum deviation from the equilibrium momentum, i.e. $d p / p$. This is mainly defined during the multiturn injection. To find out the beam $d p / p$ in the ring, the Linac beam momentum spread, the RF voltage manipulation, and the space charge effect all have to be considered.

The second factor in determining the bunch leak is the effective total $R F$ potential at the bunch edge, which is,

$$
U\left(\phi_{\ell}\right)=U_{R F}\left(\phi_{\ell}\right)-U_{S C}\left(\phi_{\ell}\right)
$$

For a normal bunch line density, say an azimuthally centered parabolic line density, the space charge potential offsets the RF potential in the bunch passage.

Using the Hamiltonian,

$$
H=\frac{|\eta| \omega_{R F}^{2}}{2 \beta^{2} E}\left(\frac{\Delta E}{\omega_{0}}\right)^{2}-\frac{e}{2 \pi} U(\phi)
$$

one obtains the relation between the total potential, combined with the $\mathrm{RF}$ and space charge, and the energy deviation. The corresponding momentum deviation is,

$$
\frac{d p}{p}=\sqrt{\frac{e U(\phi)}{\pi \beta^{2} E|\eta|}}
$$

If the maximum particle momentum deviation is larger than the one obtained using (8), at the bunch edge $\phi_{\ell}$, then a bunch leakage will happen.

An important mechanism of the space charge induced bunch lengthening is the self regulation. For the same intensity, a longer bunch will carry a 
smaller space charge potential at the bunch edge. Without loss of generality, we calculate the space charge potential using a bunch shape of cosine.

In Fig. 1 $a$, the space charge potential is compared with the RF potential, under the condition of $N=2 \times 10^{14}$ and $Z_{S C}=-j 196 \Omega$. If the half bunch length is $0.52 \pi$, then the space charge almost completely offsets the RF potential, the bunch leakage will definitely happen. The total RF potential, however, increases rapidly for the longer bunch, due to both the RF potential increase and the space charge potential decrease. In Fig. 1b, the momentum

deviation corresponding to the total RF potential is shown. At the half bunch length of $0.69 \pi$, the particles with the maximum momentum deviation smaller than $0.60 \%$ will not leak into the interbunch gap.

In the case that the particle maximum momentum deviation is larger, then the bunch leak will happen. Once the bunch lengthening occurs, the longer bunch reduces the space charge potential at the extended bunch edge. Ideally, this has a negative impact on further bunch lengthening.

Without the space charge effect, the beam maximum momentum spread can be estimated using the Hamiltonian (7). Since the space charge effect would reduce the total beam momentum spread in the injection, simulations are, therefore, needed to find out exact beam momentum spread in the ring.

\section{Simulation}

For SNS, the incoming Linac beam is chopped at the phase deviation $\pm 0.67 \pi$. The RF voltage can be $V_{R F}=40 \mathrm{KV}$ per turn. The injection takes about 1,240 turns to get $N=2 \times 10^{14}$ protons per pulse.

For the particle longitudinal motion, the following equations are used in the simulation.

$$
\frac{d}{d t}\left(\frac{\Delta E}{\omega_{0}}\right)=\frac{e}{2 \pi}\left(V_{R F}(\sin \phi-0.5 \sin 2 \phi)+V_{S C}\right)
$$

and

$$
\frac{d}{d t} \phi=\frac{\omega_{0}^{2} \eta}{\beta^{2} E}\left(\frac{\Delta E}{\omega_{0}}\right)
$$

where $\Delta E$ is the energy gain per turn. For convenience, the particle momentum deviation $d p / p$ is used in the simulation, with the following relation to the energy deviation, 


$$
\frac{d p}{p}=\frac{\omega_{0}}{\beta^{2} E}\left(\frac{\Delta E}{\omega_{0}}\right)
$$

In the simulation, homogeneously distributed Linac beam with momentum spread $d p / p= \pm 0.1 \%$ is used. In Fig. $2 a$ and $2 b$, the particle distributions without and with space charge effect are presented. With the space charge effect, the bunch half length is $0.69 \pi$. This agrees with the analytical prediction, which shows that with $V_{R F}=40 K V$, and $N=2 \times 10^{14}$, a half bunch length of $0.69 \pi$ can contain particles with the maximum momentum deviation smaller than $0.60 \%$. One may observe that in Fig. $2 b$, the maximum $d p / p$ is just a little larger than that.

Note that the synchrotron frequency is about $1 \mathrm{KHz}$ at $V_{R F}=40 \mathrm{KV}$, therefore, the entire injection process is less than one synchrotron period. This also contributes to a limited bunch length.

An immediate approach to further limit the bunch length is to utilize a ramped $R F$ voltage during the injection. For instance, to let the $R F$ voltage to ramp from $20 \mathrm{KV}$ at the start to $40 \mathrm{KV}$ at the end will define a smaller beam momentum spread in the ring. In Fig. $2 c$, the simulation result is presented. The half bunch length stays at $0.67 \pi$, and the maximum momentum spread is not larger than $0.60 \%$.

For longitudinal microwave instability, under the condition that the peak current $80 A$, Keil-Schnell stability criterion requires that $d p / p \geq 0.70 \%$. Consider that SNS is operated below transition, $d p / p \approx 0.60 \%$ is probably not a problem.

\section{Discussion}

At the PSR of LANL, the bunch leakage into the interbunch gap is suspected to be responsible to the e-p instability. With the same simulation scheme, the increasing intensity caused bunch lengthening due to the longitudinal space charge is shown in Fig.3. The $\mathrm{RF}$ voltage is $6 \mathrm{KV}$ and the Linac beam momentum spread is $d p / p= \pm 0.1 \%$, chopped at the phase deviation $\pm 0.70 \pi$. It can be noticed that the space charge induced bunch leakage becomes significant at $N=3 \times 10^{13}$, causing a half bunch length 0.96 $\pi$. Meanwhile, the beam momentum spread in the ring constantly decreases along with the increase of the intensity, presumably due to the longitudinal space charge effect. 
For the best possible injection scheme at the PSR, the $\mathrm{RF}$ voltage ramping from $6 \mathrm{KV}$ at the start to $10 \mathrm{KV}$ at the end is utilized to give rise to half bunch length of $0.74 \pi$, obtained using the same simulation. This leakage is sizable, but is not strong enough to stimulate the e-p instability. In [1], a possible beam loading effect is speculated as an even stronger mechanism of the bunch leakage.

To combat with the longitudinal space charge effect, a correction scheme by inserting ferrite in the ring is studied at the PSR [2]. An improvement of the bunch leakage was observed. At the SNS, it seems that based on the analytical and simulation results, the bunch leakage due to the longitudinal space charge is limited. Therefore, a similar correction is probably not necessary.

As for the beam momentum distribution in the ring, to use the Linac beam with momentum spread $d p / p= \pm 0.1 \%$ is probably not the best choice. In Figs. 4 and 5, the simulation results are shown for the 2 MW SNS injection with and without RF ramping, respectively, for different Linac beam momentum spread. The bunch lengthening is still tolerable at $d p / p \geq 0.2 \%$, but the beam momentum distribution is improved. 


\section{References}

${ }^{1}$ S.Y. Zhang and W.T. Weng., 'Beam Loading Effect in Bunch Leakage at the $P S R$,' submitted for publication.

${ }^{2}$ J.E. Griffin et. al., 'Experimental Study of Passive Compensation of Space Charge Potential Well Distortion at the PSR,' Fermilab, FN-66I, Nov. 1997. 

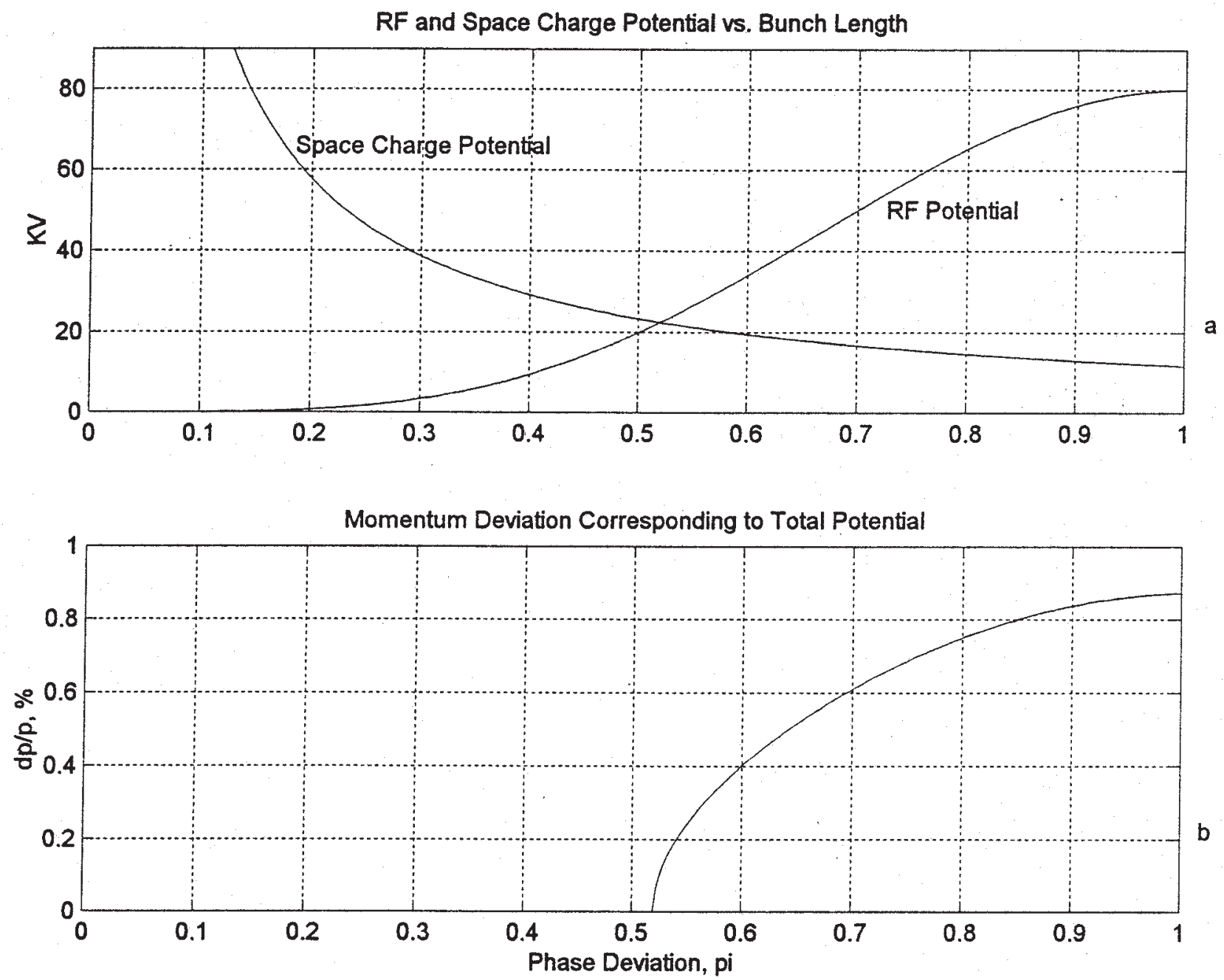

Fig.1. SNS RF and Space Charge Potential 

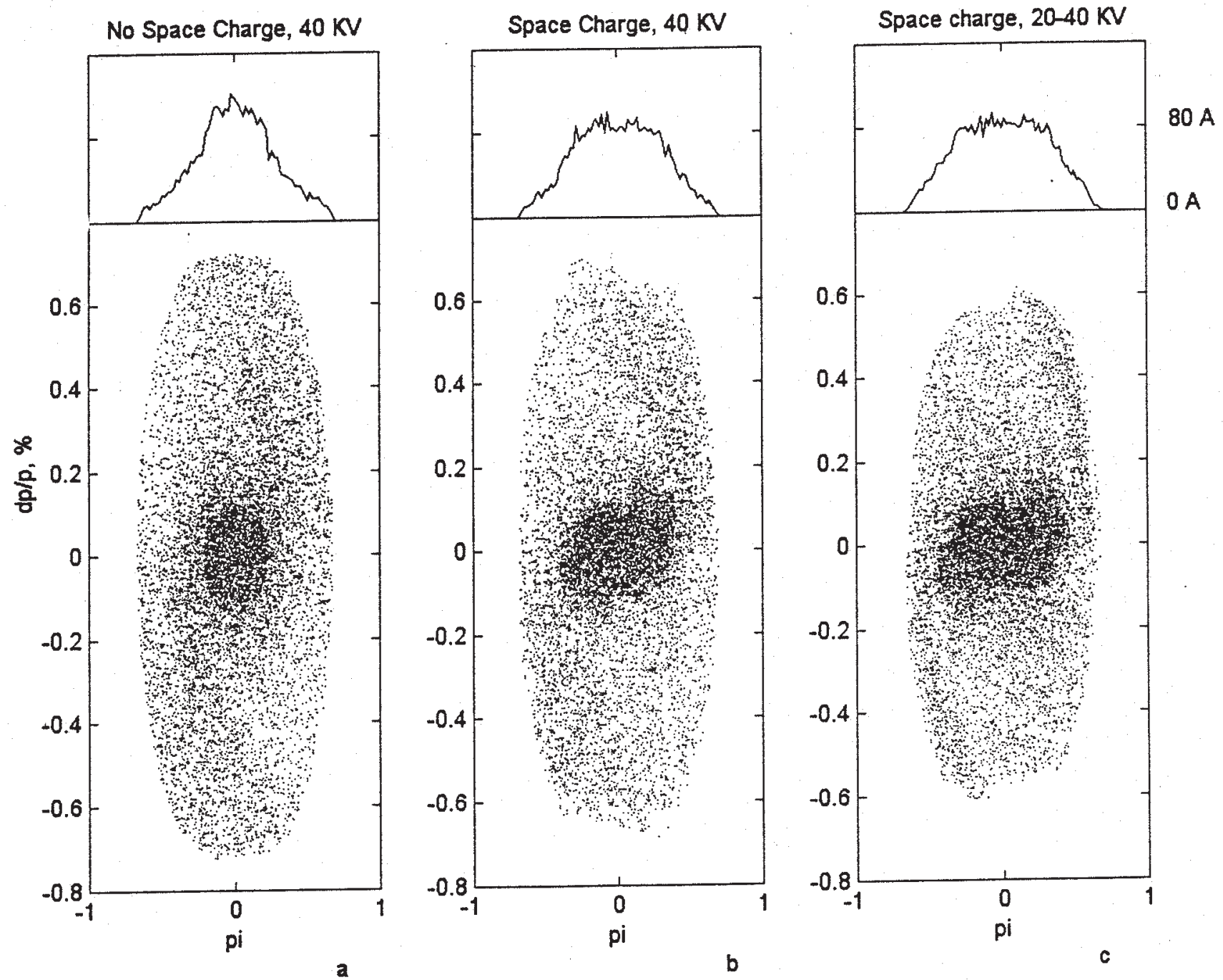

Fig.2. SNS Longitudinal Space Charge effect, Half Bunch Length: a: $0.67 \pi$, b: $0.69 \pi$, c: $0.67 \pi$ 


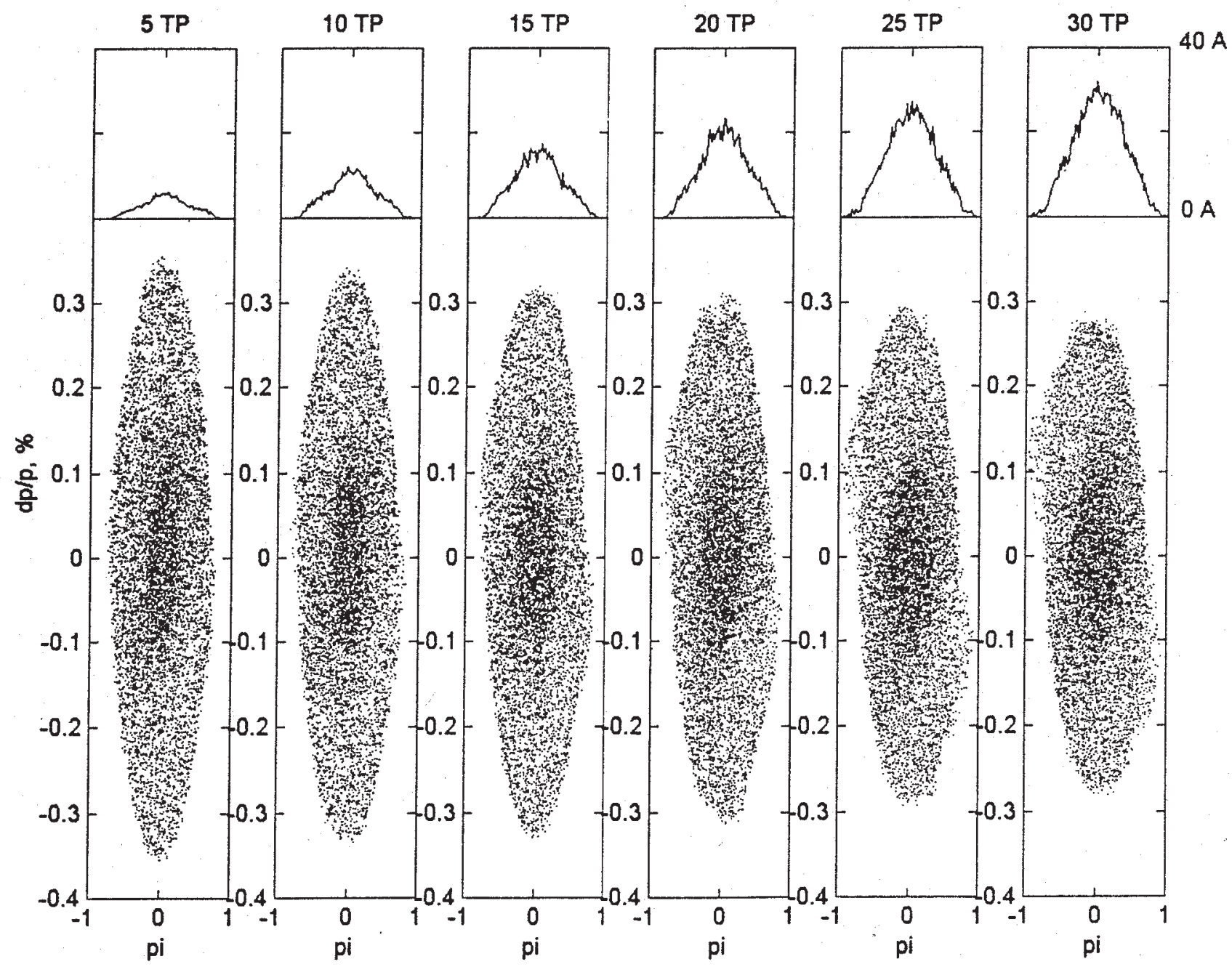

Fig.3. PSR Longitudinal Space Charge effect with $6 K V$ RF Voltage 

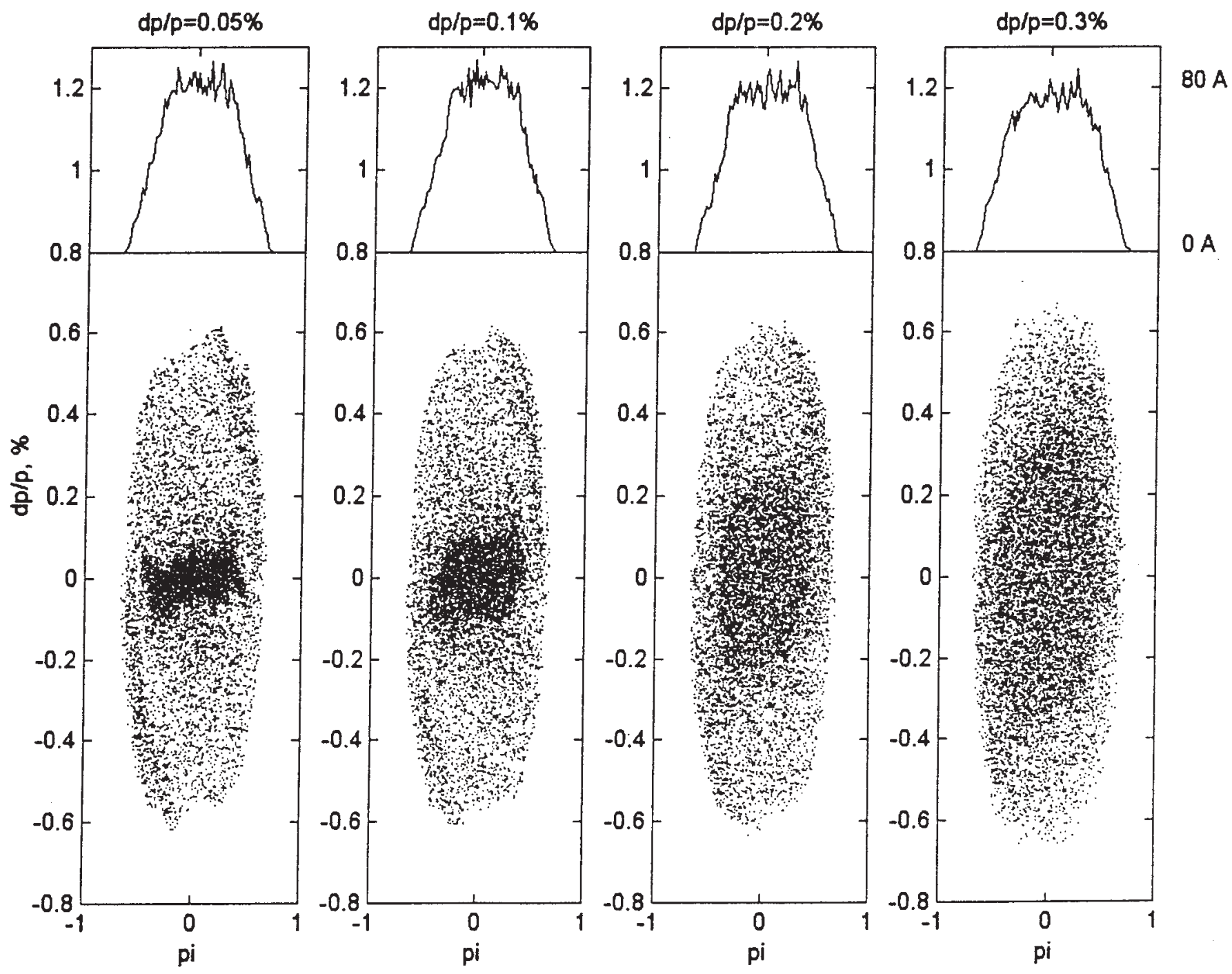

Fig.4. SNS Longitudinal Space Charge effect, With RF Voltage Ramping,

Half Bunch Length: $0.67 \pi, 0.67 \pi, 0.67 \pi, 0.70 \pi$ 

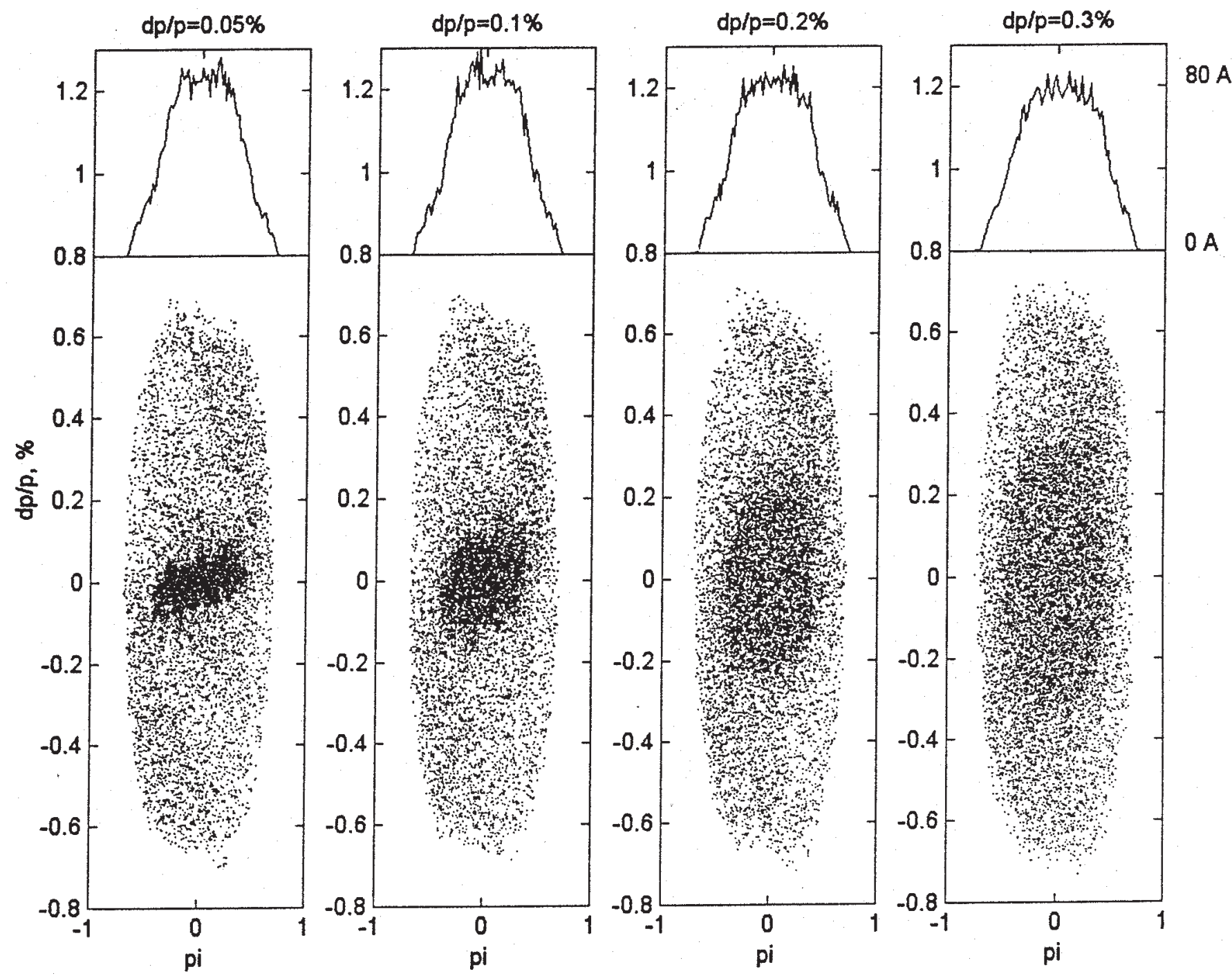

Fig.5. SNS Longitudinal Space Charge effect, Without RF Voltage Ramping,

Half Bunch Length: $0.69 \pi, 0.69 \pi, 0.72 \pi, 0.74 \pi$ 